\title{
BMJ Open Little in Norway: a prospective longitudinal community-based cohort from pregnancy to child age 18 months
}

\author{
Vibeke Moe (1) , ${ }^{1,2}$ Eivor Fredriksen, ${ }^{1,2}$ Marian Kjellevold, ${ }^{3}$ Lisbeth Dahl, ${ }^{3}$ \\ Maria Wik Markhus, ${ }^{3}$ Kjell Morten Stormark, ${ }^{4}$ Tilmann von Soest, ${ }^{1}$ \\ Kåre Sten Olafsen, ${ }^{2}$ Unni Tranaas Vannebo, ${ }^{2}$ Lars Smith ${ }^{1}$
}

To cite: Moe V, Fredriksen E, Kjellevold M, et al. Little in Norway: a prospective longitudinal community-based cohort from pregnancy to child age 18 months. BMJ Open 2019;9:e031050. doi:10.1136/ bmjopen-2019-031050

- Prepublication history for this paper is available online. To view these files, please visit the journal online (http://dx.doi. org/10.1136/bmjopen-2019031050).

Received 15 April 2019

Revised 14 November 2019 Accepted 20 November 2019

Check for updates

(C) Author(s) (or their employer(s)) 2019. Re-use permitted under CC BY-NC. No commercial re-use. See rights and permissions. Published by BMJ.

${ }^{1}$ Department of Psychology, Faculty of Social Sciences, University of Oslo, Oslo, Norway ${ }^{2}$ Regional Centre for Child and Adolescent Mental Health,

East and South, Norway, Oslo, Norway

${ }^{3}$ Institute of Marine Research, Bergen, Norway

${ }^{4}$ Centre for Child and Adolescent Mental Health Uni Health, Uni Research, Bergen, Norway

Correspondence to

Dr Vibeke Moe;

vibeke.moe@psykologi.uio.no

\section{ABSTRACT}

Purpose The Little in Norway (LiN) project is a crossdisciplinary prospective longitudinal study starting in pregnancy. It was set up to investigate maternal and paternal mental health functioning in the transition to parenthood, detect pathways to healthy and aberrant child development and generate new knowledge about mechanisms underlying differential child mental health susceptibility.

Participants The LiN cohort is a community-based sample comprising 1036 families (1036 mothers, 884 partners, 1017 children). All pregnant women and their partners receiving routine prenatal care at well-baby clinics at nine geographically selected sites across Norway were invited to participate. Enrolment took place from September 2011 to October 2012. This cohort profile comprises 10 data collection waves spanning from enrolment in pregnancy until child age 18 months.

Findings to date Four types of information have been collected: multi-informant questionnaire reports, direct observation of interaction, test data and biological samples. The most significant findings so far relate to three domains of results. First, when examining risk factors for parental mental health problems, results showed that the parents' own adverse childhood experiences and attachment style were related to anxiety, depression and stress in the perinatal period. The perception of difficult child temperament was also found to contribute to parenting stress in the first year after birth. Second, we studied how parental mental health risk factors were related to later child development and social emotional functioning, for example, linking maternal symptoms to socialemotional outcomes and paternal symptoms to language outcomes. Third, we investigated the relation between maternal nutrition during pregnancy and aspects of early child development. Results showed that mild to moderate maternal iodine deficiency in pregnancy was associated with poorer language skills up to 18 months, but not with reduced cognitive or fine and gross motor skills.

Future plans A data collection point at 36 months is completed and currently being analysed. A new data collection wave is planned when the children are 8 years of age.

Trial registration number ISRCTN66710572.
Strengths and limitations of this study

- Cross-disciplinary prospective longitudinal community-based study of children and their parents from early pregnancy on.

- A multimethod and multi-informant design including biological sampling, direct observation of behaviour, assessment of the children's development and selfreported information from mothers and fathers.

- Many repeated in-depth assessments from early in pregnancy and onward make it possible to identify differential developmental pathways and underlying mechanisms.

- This cohort alone may be underpowered when examining risk factors of outcomes with low prevalence, hence collaboration is encouraged.

- The participating parents tended to have a higher educational level than the general population at the different sites.

- A related concern is selective attrition, as dropout was shown to be related to depressive symptoms.

- Appropriate statistical methods have been used to minimise the effect of selective attrition in papers based on this study population.

\section{INTRODUCTION}

Differential pathways to healthy and atypical development emerge early in life. ${ }^{1}$ Human infants are malleable and take their course of growth in many directions, in part predisposed by biological factors and subsequently influenced by postnatal environmental characteristics in a continuous dynamic transactional interplay. ${ }^{2-4}$ Such pathways have been identified in early childhood. ${ }^{256}$ However, the knowledge of the prenatal and perinatal precursors of child development and socialemotional functioning is still scarce. The Little in Norway (LiN) study is an ongoing cross-disciplinary longitudinal and prospective multisite community-based study of children and their parents from early pregnancy on. It was set up to investigate maternal and paternal mental health functioning in the 
transition to parenthood, examine prenatal and postnatal precursors of long-term child development and mental health, as well as disentangling mechanisms of transmission of developmental risk and protective factors. The study aims at integrating the research areas of mental health, developmental psychology, nutrition and molecular genetics. This cohort profile focuses on 10 data collection waves spanning from enrolment in pregnancy until 18 months after birth.

A better understanding of early risk and protective factors, leading into pathways of later competence or adversity is needed, as there is a high potential for early interventions to adjust atypical pathways. ${ }^{7-9}$ For example, parents' adverse childhood experiences ${ }^{10}{ }^{11}$ have been shown to influence later parental mental health, and are associated with insecure attachment, prenatal and postnatal depression, parenting stress and atypical parenting behaviour towards their own children. ${ }^{10} 12-17$ However, only few studies have examined the longitudinal implications of such risk factors on parenting and parentchild interaction in cohorts followed from the prenatal period. ${ }^{18}$ One needs to elucidate the mechanisms of how parental adverse childhood experiences and mental health status, such as perinatal depression and anxiety, translate into aberrant child development, as such mechanisms are only partially understood. ${ }^{19}{ }^{20}$ In addition, there is a need for including fathers in such studies. ${ }^{2122}$ Particularly, there is a lack of studies examining differential and joint effects of mothers' and fathers' impact on mental health functioning in infancy and early childhood. ${ }^{23}$

Antenatal biological factors may also be decisive for later development. Some nutrients protect maternal health while others affect birth outcome and infant health. For example, an inadequate nutrient supply may cause biological competition between mother and fetus. ${ }^{24}{ }^{25}$ Evidence also suggests that long chain polyunsaturated fatty acids, such as docosahexaenoic acid (DHA, 22:6, n3), play a role in the aetiology of postpartum depression. ${ }^{26-29}$ DHA and arachidonic acid (AA, 20:4, n-6) are important structural components in the brain and the central nervous system and subsequently necessary for normal growth and development of the brain. Furthermore, suboptimal iodine nutrition during pregnancy is associated with poorer child language development and lower child IQ. ${ }^{30-32}$ This study comprises a Food Frequency Questionnaire applied at several time points as well as biological samples to obtain information related to child and parental nutrition and its possible relation to child development and mental health functioning.

\section{COHORT DESCRIPTION}

\section{Who is in the cohort?}

All pregnant women, receiving routine prenatal care at nine public well-baby clinics across Norway, were invited by midwives to participate. The clinics were chosen taking demographics and size of the population into account, to ensure a wide distribution of background conditions.
Participants were recruited at their first prenatal care examination at the well-baby clinic. At each site, one public healthcare nurse was trained as a research assistant. In Norway, all pregnant women have the right to prenatal care and at least eight free consultations during pregnancy. If necessary, extra consultations are offered. Nearly all pregnant women (93\%) in Norway choose to use the first-line public healthcare system during pregnancy (general practitioners and well-baby clinics). ${ }^{33}$ Although there are nationwide schedules for prenatal care, many choose to receive initial check-ups by their general practitioner while switching to midwives at the well-baby clinics when the due date approaches. Therefore, despite all women being invited at their first prenatal visit to the well-baby clinic, the time frame for enrolment ranged from gestational week 8 to 34 .

Initially, 1041 women consented; 5 later withdrew, leaving 1036 (99.5\%) participating pregnant women. Their partners were also invited and 884 (878 men and 6 women) participated. No exclusion criteria were applied, but since the questionnaires were either in Norwegian or in English, this might have excluded pregnant women and their partners who spoke neither Norwegian nor English. The participation rate is estimated to be $50.7 \%$. At five of the clinics, the staff did not establish reliable routines to monitor rates of participation; this rate is based on records from the remaining four clinics. However, participation rates were probably relatively similar at all sites since all clinics shared recruitment strategies.

Table 1 shows demographic characteristics of the sample. Pearson's $\chi^{2}$ tests were performed at each of the nine sites separately, comparing the participants' educational level with the mean educational level of the population at the nine sites in the same age range (figures obtained from Statistics Norway). In general, participants showed higher educational level than the reference populations $(\mathrm{p}<0.001)$.

Participants received written and oral information before giving their consent to their own and their children's participation. Confidentiality was assured, and it was emphasised that they could withdraw at any time. The enrolment took place from September 2011 to October 2012. At time of birth, 3 families were lost due to stillbirth, while 26 families were lost for other reasons before childbirth (eg, family moving out of the area, participation felt to be too time consuming). The remaining 1007 mothers delivered 1017 children (including 10 twin pairs).

\section{How often have the participants been followed up?}

The participants have been followed-up from early in pregnancy to infant age 18 months. The study has an intensive prospective, longitudinal design including data collection at 10 time points. After enrolment, the prenatal data collection waves were at gestational weeks 8-21 (T1), weeks 22-27 (T2), weeks 28-31 (T3), weeks 32-35 (T4) and weeks 36-41 (T5). T6 comprises birth records. Postnatal data were collected at 6 weeks post partum (T7), 
Table 1 Demographic characteristics of participants in the LiN study

\begin{tabular}{|c|c|c|c|}
\hline & $\begin{array}{l}\text { Mothers } \\
\mathrm{n}=1036 \\
\text { Mean (SD)/ } \\
\text { proportion }\end{array}$ & $\begin{array}{l}\text { Partners } \\
\mathrm{n}=884 \text { (878 } \\
\text { men) } \\
\text { Mean (SD)/ } \\
\text { proportion }\end{array}$ & $\begin{array}{l}\text { Children } \\
\mathrm{n}=1017 \\
\text { Mean (SD)/ } \\
\text { proportion }\end{array}$ \\
\hline \multicolumn{4}{|l|}{ Parents } \\
\hline Age (years) & $30.26(4.78)$ & $32.76(5.90)$ & \\
\hline Education (years) & $16.05(2.13)$ & $15.59(2.37)$ & \\
\hline College or university degree & $77.1 \%$ & $67.1 \%$ & \\
\hline High school & $19.8 \%$ & $28.2 \%$ & \\
\hline Elementary school & $3.1 \%$ & $4.8 \%$ & \\
\hline Ethnic minority & $6.1 \%$ & $4.6 \%$ & \\
\hline First-time parent & $54.9 \%$ & $56.2 \%$ & \\
\hline One previous child & $33.3 \%$ & $32.7 \%$ & \\
\hline Two or more previous children & $11.8 \%$ & $11.0 \%$ & \\
\hline \multicolumn{4}{|l|}{ Work status } \\
\hline Full-time job & $77.3 \%$ & $91.0 \%$ & \\
\hline Part-time job & $7.4 \%$ & $1.7 \%$ & \\
\hline Student & $11.6 \%$ & $6.2 \%$ & \\
\hline $\begin{array}{l}\text { Disability/Unemployed/At } \\
\text { home }\end{array}$ & $3.8 \%$ & $1.0 \%$ & \\
\hline \multicolumn{4}{|l|}{ Relationship status } \\
\hline Married & $36.2 \%$ & $35.2 \%$ & \\
\hline Living together & $59.7 \%$ & $62.4 \%$ & \\
\hline Single & $2.5 \%$ & $0.9 \%$ & \\
\hline Divorced & $0.2 \%$ & $0.2 \%$ & \\
\hline Other & $1.4 \%$ & $1.2 \%$ & \\
\hline Previous psychiatric problems & $21.7 \%$ & $11.2 \%$ & \\
\hline Life stress at enrolment & $7.08(6.91)$ & $7.38(7.00)$ & \\
\hline \multicolumn{4}{|l|}{ Children } \\
\hline Gestational age (in weeks) & & & $39.99(1.81)$ \\
\hline Sex (percentage of boys) & & & $52.3 \%$ \\
\hline Premature births & & & $6.3 \%$ \\
\hline
\end{tabular}

LiN, Little in Norway.

and again at 6 months (T8), 12 months (T9) and 18 months (T10) after birth, all within \pm 2 weeks.

All participants completed the enrolment package ( $\mathrm{n}=1036$ and 884, pregnant women and their partners, respectively). Data collection at T1-T10 had limited time windows relative to gestational/child age for valid participation. This means that a sizeable proportion of participants was not enrolled in time to take part in the early data collection points during pregnancy. At T10, 925 families $(88.9 \%)$ of the original sample were still participants and remained in the study for future follow-up. Hence, there are three categories of missing data: late recruitment, study withdrawal or families lost to follow-up $(n=116)$ and intermittently missing data, which comprise participants that skipped one or more data collection waves, but then contributed at later time points. Figure 1 depicts the flow of recruitment, participation and attrition at each data point.
Bivariate logistic regression analyses were conducted to investigate selective attrition at T10 (18 months after childbirth). High level of maternal education slightly increased the probability of participation at $\mathrm{T} 10(\mathrm{OR}=1.07,95 \% \mathrm{CI}$ 1.01 to $1.15, \mathrm{p}=0.030)$, measured in years of education. Neither partners' education ( $\mathrm{OR}=1.04,95 \% \mathrm{CI} 0.97$ to $1.11, \mathrm{p}=0.255)$, maternal age $(\mathrm{OR}=1.00,95 \% \mathrm{CI} 0.97$ to $1.03, \mathrm{p}=0.886)$, partners' age $(\mathrm{OR}=1.00,95 \% \mathrm{CI} 0.98$ to 1.03, $\mathrm{p}=0.756$ ), number of previous children for mothers ( $\mathrm{OR}=0.87,95 \%$ CI 0.73 to $1.04, \mathrm{p}=0.123)$, nor the number of partners' previous children (OR=0.98, 95\% CI 0.80 to $1.21, \mathrm{p}=0.879$ ) were related to attrition. Attrition was further predicted by lower levels of mean prenatal $(\mathrm{OR}=0.91,95 \%$ CI 0.88 to $0.95, \mathrm{p}<0.001)$ and postnatal $(\mathrm{OR}=0.95,95 \%$ CI 0.90 to $1.00, \mathrm{p}=0.041)$ depressive symptoms for mothers, as well as lower levels of mean prenatal $(\mathrm{OR}=0.92,95 \% \mathrm{CI} 0.87$ to $0.97, \mathrm{p}=0.004)$ and postnatal ( $\mathrm{OR}=0.93,95 \%$ CI 0.86 to $0.99, \mathrm{p}=0.030)$ depressive symptoms for partners. Depressive symptoms were measured by the composite scores of Edinburgh Postnatal Depression Scale (EPDS). ${ }^{34}$

\section{What has been measured?}

Data collected in the LiN study include multi-informant questionnaire data, observational and behavioural data and biological samples. Data were collected at the local well-baby clinic and by means of web-based surveys filled out at home, at well-baby clinics or completed by the research assistants based on parent interviews. Types of measurements at each wave are shown in table 2, while the specific measurement methods are shown in table 3 .

\section{Enrolment package in pregnancy}

Mothers and fathers received a comprehensive questionnaire package concerning parental demographic information, somatic and mental health and dietary habits, as well as medication, smoking and alcohol habits, at the first meeting in pregnancy (enrolment package). To screen for possible alcohol problems the Tolerance, Worried, Eyeopener, Amnesia, Cut-down screening questionnaire ${ }^{35}$ was administered. In addition, possible adverse childhood experiences were assessed by means of the Adverse Childhood Experiences Scale. ${ }^{10}$ The scale comprises 10 questions about possible adverse experiences prior to age 18 years, such as physical or sexual assault, major separation or loss or parental mental illness or drug abuse. The Life Stress Scale, a subscale of the Parenting Stress Index (PSI) ${ }^{36}$ was used to measure life stress events. The PSI is a self-report questionnaire that contains a Life stress Scale, a Child Domain, reflecting how parents perceive their child, and a Parent Domain, consisting of items related to parental coping and the parenting role. The PSI was standardised for use with parents of children ranging from 1 month to 12 years of age. The Life stress Scale consists of 19 items measuring life stress events experienced in the previous 12 months.

We also measured partner-related attachment style by using the Experiences in Close Relationships (ECR) 


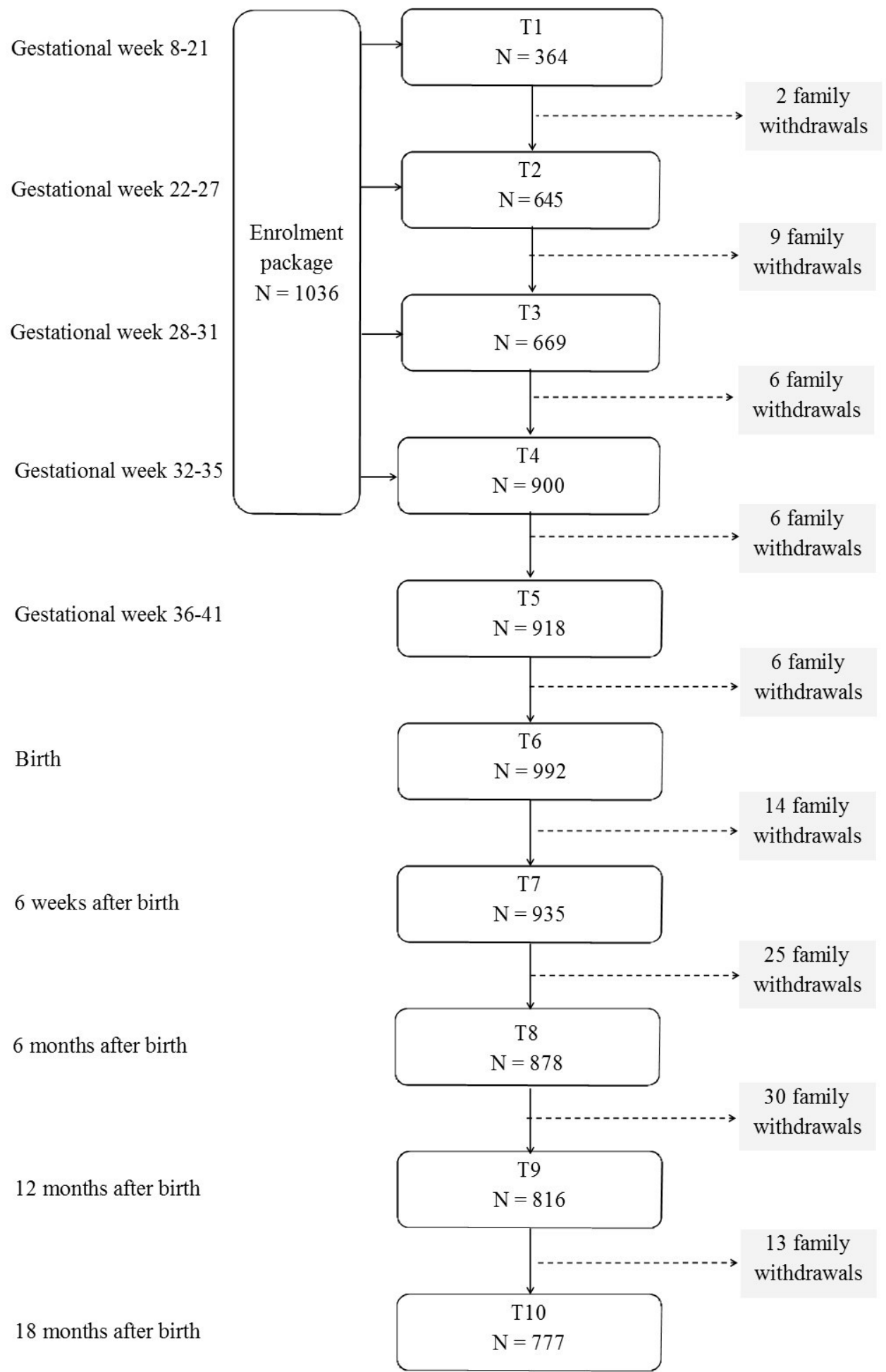

Figure 1 Recruitment, participation and attrition in the Little in Norway study. All participants received an enrolment package at entry into the study; subsequently, they took part in the data collection wave corresponding to their gestational week at entry. Due to late recruitment, some participants missed early data collection waves and the full Little in Norway sample was reached at T4. The Ns represent the number of participants taking part at each time point; missingness comprises late recruitment, intermittent missingness (missing at current time point, but participating at later time points) and study dropout. The grey boxes on the right indicate the number of participants that dropped out on a permanent basis.

Scale $^{37}$ since the quality of the parents' representations of attachment relationships is an important factor to consider for parental adaptation and parenting behaviour. $^{38}{ }^{39}$ The ECR Scale is a self-report measure designed to assess the dimensions of adult attachment styles in relationships. The instrument comprises two dimensions, avoidance and anxiety, consisting of 18 items each. 


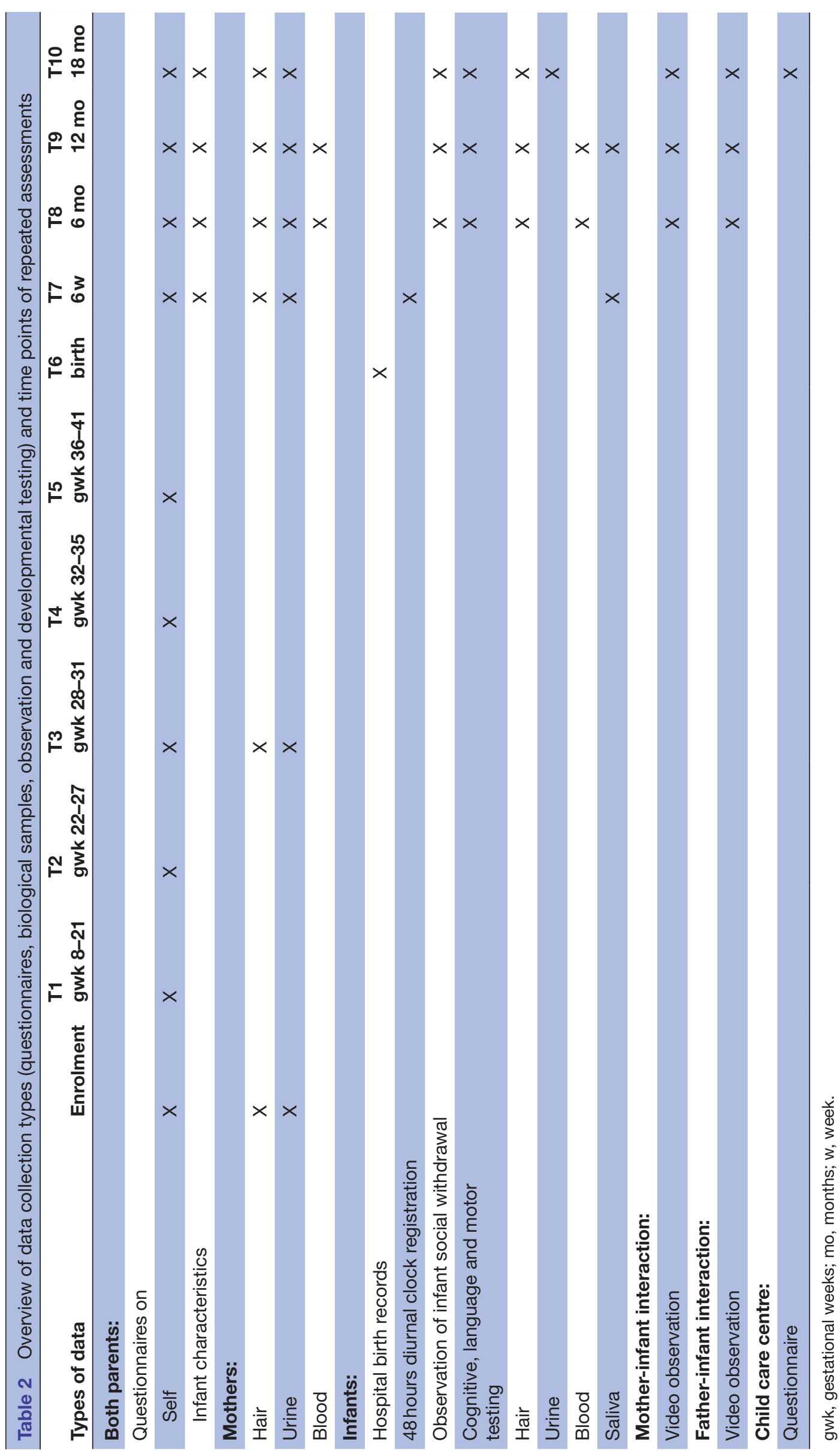

\begin{tabular}{l} 
س \\
3 \\
\hdashline \\
0 \\
0
\end{tabular} 
Table 3 Phases of data collection and specific measurements in the LiN study

\begin{tabular}{|c|c|}
\hline Phase & Measurements \\
\hline Assessments during pregnancy & Enrolment package, T1 (gwk 8-21), T2 (gwk 22-27), T3 (gwk 28-31), T4 (gwk 32-35), T5 (gwk 36-41) \\
\hline $\begin{array}{l}\text { Enrolment package in pregnancy, first } \\
\text { visit to well-baby clinic (range gwk } \\
\text { 8-34) } \\
\text { At well-baby clinic }\end{array}$ & $\begin{array}{l}\text { Mothers and fathers: demographic information, somatic and mental health, medication/smoking/ } \\
\text { alcohol habits (TWEAK), thoughts about unborn child, life stress (PSI-LS), relational experiences } \\
\text { (ECR), adverse childhood experiences (ACE) } \\
\text { Mothers only: FFQ*, hair and urine samples }\end{array}$ \\
\hline
\end{tabular}

Web from home*

T1 (gwk 8-21) Both parents: pregnancy anxiety concerns (PRAQ-R), depressive symptoms (EPDS), medication/ smoking/alcohol habits (TWEAK), thoughts about unborn child Mothers only: brief FFQ

T2 (gwk 22-27)

Web from home

Both parents: pregnancy anxiety concerns (PRAQ-R), medication/smoking/alcohol habits (TWEAK), depressive symptoms (EPDS), thoughts about unborn child Mothers only: brief FFQ

T3 (gwk 28-31)

At well-baby clinic

T4 (gwk 32-35)

Web from home
Both parents: pregnancy anxiety concerns (PRAQ-R), medication/smoking/alcohol habits (TWEAK), depressive symptoms (EPDS), thoughts about unborn child Mothers only: brief FFQ, blood, urine and hair samples

Both parents: pregnancy anxiety concerns (PRAQ-R), medication/smoking/alcohol habits (TWEAK), depressive symptoms (EPDS), thoughts about unborn child Mothers only: FFQ

T5 (gwk 36-41)

Both parents: pregnancy anxiety concerns (PRAQ-R), medication/smoking/alcohol habits (TWEAK), depressive symptoms (EPDS), thoughts about unborn child Mothers only: brief FFQ

\section{T6 birth}

Birth weight; gestational age; birth complications

Follow-up

At well-baby clinic

Web from home*

T7 child age 6 weeks

At well-baby clinic

Web from home*

T7 6 weeks, T8 6 months, T9 12 months, T10 18 months

Infant-mother assessment procedure (MABI)

Infants: diurnal rhythm; DNA extracted from saliva, infant hair samples

Mothers: FFQ*, child nutrition and breast feeding*, maternal hair and urine samples

Both parents: depressive symptoms (EPDS)

T8 child age 6 months

At well-baby clinic

Web from home*

Infants: infant development (Bayley Scales, screening test + ); infant social withdrawal behaviour (ADBB); hair and blood samples

Mothers: hair, urine and blood samples, FFQ*, child nutrition and breast feeding*

Both parents: PSI, infant-parent interaction, depressive symptoms (EPDS), perceived infant temperament* (Cameron-Riceł)

T9 child age 12 months

At well-baby clinic

Web from home*

Infants: infant development (Bayley Scales, screening test + ), infant social withdrawal behaviour (ADBB), genetic information extracted from saliva, infant hair and blood samples

Mothers: FFQ*, child nutrition and breast feeding ${ }^{*}$, maternal hair, urine and blood samples Both parents: PSI, infant-parent interaction, depressive symptoms (EPDS), perceived infant temperament* (Cameron-Rice $\neq)$

T10 child age 18 months

At well-baby clinic

Web from home*

Web from child care centres $\Uparrow$
Infants: infant development (Bayley Scales, full scale§), infant social withdrawal behaviour (ADBB), infant hair and urine samples

Mothers: maternal hair and urine samples

Both parents: PSI, infant-parent interaction, depressive symptoms (EPDS), child social emotional assessment* (ITSEA)

Childcare centre: child social emotional assessmentๆ (ITSEA)

*Web from home.

†Bayley Scales of Infant and Toddler Development, screening test. ${ }^{46}$

$\ddagger$ Cameron-Rice perceived temperament scale. ${ }^{48}$

§Bayley Scales of Infant and Toddler Development, full scale. ${ }^{46}$

ПWeb from child care centres.

ACE, Adverse Childhood Experiences Scale ${ }^{10}$; ADBB, Alarm Distress Baby Scale ${ }^{47}$; ECR, Experiences in Close Relationships Scale ${ }^{37}$; EPDS,

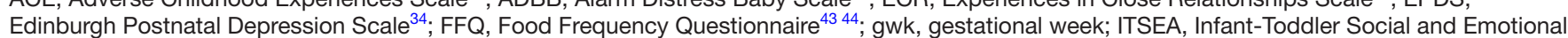

Assessment ${ }^{50}$; MABI, Mothers's Assessment of the Behaviour of the Infant ${ }^{45}$; PRAQ-R, Pregnancy-Related Anxiety Questionnaire-Revised ${ }^{40}$; PSI,

Parenting Stress Index ${ }^{36}$; PSI-LS, Parenting Stress Index-Life Stress ${ }^{36}$; TWEAK, Tolerance, Worried, Eye-opener, Amnesia, Cut-down.

Repeated assessments during pregnancy (T1-T5)

Anxiety related to pregnancy and birth and depressive symptoms in the perinatal period were measured at all assessments in pregnancy in both parents (T1-T5). Pregnancy-Related Anxiety Questionnaire ${ }^{40}$ is a 10 -item scale designed to assess ongoing anxiety related to pregnancy and birth. The Edinburgh Postnatal Depression Scale (EPDS) ${ }^{34}$ is a self-report measure to identify women at risk for postnatal depression in the previous 7 days. Although EPDS was originally developed to screen for 
postnatal depressive symptoms in women, it has later been validated for prenatal use. ${ }^{41}$ It has also been validated on male populations. ${ }^{42}$ We also included questions about both parents' thoughts and feelings towards their unborn baby at all data points during pregnancy in order to capture mental representations of the coming child. To assess nutritional status and cortisol levels, hair and urine samples were collected from the mothers twice during pregnancy (T1 and T3). The urine samples have been analysed for iodine concentration. Blood samples were collected during pregnancy (T1) and have been analysed for fatty acid composition and vitamin $\mathrm{D}$ status $(25(\mathrm{OH}) \mathrm{D} 3)$. Dietary intake was measured using a webbased Food Frequency Questionnaire twice during pregnancy (T1 and T4). ${ }^{43} 44$

Three questions about seafood intake were included at T2, T3 and T5.

\section{Postnatal assessments (T6-T10)}

At birth (T6), we collected information from hospital birth records. At 6 weeks after birth (T7), the research assistants met with the parents and their baby to observe the infant ${ }^{45}$ and obtain information about the child's diurnal rhythm. The parents received questionnaires, and biological samples from mother and child were collected.

Depressive symptoms ${ }^{34}$ in both parents were again assessed (T7-T10 by means of the EPDS. Stress in the parenting role and in the parent-child relationship was also measured repeatedly post partum by using the PSI. ${ }^{36}$ Testing and observation of the children's development and videotaping of child-parent interaction were conducted at the local well-baby clinics at 6,12 and 18 months (T8-T10). Developmental skills were assessed by using the screening ( 6 and 12 months) and the full version (18 months) of the Bayley Scales of Infant and Toddler Development, third edition. ${ }^{46}$ The Bayley Scales is an individually administered test designed to assess developmental functioning of infants and toddlers from 1 to 42 months. The screener is composed of selected items from the full Bayley version to briefly assess current functioning in the domains of cognition, receptive and expressive language, as well as gross and fine motor development. We also observed the infants' possible social withdrawal reactions using the Alarm Distress Baby Scale. ${ }^{47}$ The scale is a method aimed at evaluating social behaviours that can be observed during a brief observation of children aged from 2 to 24 months. It was scored based on child behaviour when doing the Bayley.

The parents were also asked to evaluate their infants' temperament characteristics by means of the CameronRice perceived temperament scale (CRITQ).$^{48}$ The CRITQ comprises 46 items probing eight dimensions: sensitivity, movement, reactivity/intensity, persistence, adaptability, approach-withdrawal, regularity and soothability.

To assess the quality of parent-child interaction (data not yet analysed), the children and their parents participated in videotaped play sessions at 6,12 and 18 months
(T8-T10). At 18 months (T10), the Infant-Toddler Social Emotional Assessment scale (ITSEA) ${ }^{50}$ was completed by all parents, and by the preschool teacher if the child attended a day-care centre. The ITSEA is a parent report questionnaire assessing the child's social-emotional functioning in children from 12 to 36 months and comprises 166 items measuring four domains: externalising behaviour, internalising behaviour, dysregulation and competence.

In one subsample ( $\mathrm{n}=102,10.03 \%)$, children with highrisk $(n=52)$ versus low-risk scores $(n=50)$ on markers of developmental status and parenting stress obtained at 6 months were selected. Child social emotional functioning was assessed by a standardised telephone interview with the main caregiver at 12 months (T9). ${ }^{51}$ The aim was to assess the applicability of the ITSEA at its lowest age level (12 months), and whether infants at risk at 6 months had increased scores later (12 months).

In another subsample, the aim was to investigate if changes in toddlers' morning to mid-afternoon levels of cortisol were different on days spent in childcare compared with days spent at home. ${ }^{52}$ The children's stress levels were assessed 5-6 months after they entered childcare by measuring levels of cortisol in saliva at home and in childcare. Childcare quality was observed on a day when researchers visited the childcare centres in the same period as saliva was collected. From the whole sample, 459 eligible parents who had toddlers entering childcare in the autumn of 2013 and 2014 were invited to participate (192 parents consented, 122 childcare centres cooperated). Ten children missed saliva samples, thus the final sample comprised 112 children $(58.30 \%$ of the consenting parents, $11.01 \%$ of the total child sample).

Dietary intake was assessed repeatedly post partum, both for mothers and infants. ${ }^{43}{ }^{44}$ Hair samples and nonfasting urine samples from the mothers were collected at 6 weeks, 6, 12 and 18 months post partum (T7-T10), and non-fasting venous blood samples were collected at 6 and 12 months post partum (T8-T9). The blood samples have been analysed for fatty acid composition, thyroid hormone function and 25(OH)D3 status. Non-fasting blood samples were collected from the child at 6 and 12 months post partum (T8-T9). Hair from the child was collected at 6 weeks, 6,12 and 18 months post partum (T7-T10) and spot urine samples were collected at 18 months (T10). The urine samples have been analysed for iodine concentration, and a subsample of hair (T7) has been analysed for total mercury concentration. The blood samples have been analysed for fatty acid composition and 25(OH)D3 status. Saliva samples, to be used for assaying DNA-methylation, were collected at 6 weeks (T7) and 12 months (T9). It should be noted that at age 3 years information about the children's social emotional functioning at home and (when applicable) in day care, as well as an update on the families' living conditions were collected.

The next data collection wave is planned when the children are 8 years of age. The main aim of the 8-year 
longitudinal follow-up study will be to investigate a broad range of child outcomes, including mental health and social-emotional and cognitive functioning, during the key transitional phase of entering school, predicted by perinatal, psychological, epigenetic and nutritional patterns and processes. We have so far used a genomewide epigenetic approach, which allows a non-biassed screen for DNA methylation alterations that may be associated with prenatal maternal stress. ${ }^{53}$ We will pursue this line of investigation in future studies. We plan to look at patterns of parental mental health and non-optimal nutrition, and possible relations to differential child methylation patterns. Subsequent collection waves are planned at age 13 and later on.

\section{PATIENT AND PUBLIC INVOLVEMENT STATEMENT}

The LiN cohort is population based and does not involve patients. However, all four Regional Centres for Child and Adolescent Health in Norway were involved in the planning and development of the design of the study, and the data collection involved collaboration with midwives and nurses from nine public well-baby clinics across the four different Norwegian health regions.

\section{Findings to date}

The most important findings generated by the cohort so far can be summarised in three main domains. The first domain concerns parental mental health functioning in the perinatal period and factors related to parental anxiety, depression and stress in mothers and fathers. 18225455 The second domain is directed towards child functioning and early detection of infants and toddlers at risk, ${ }^{23515256}$ while the third domain concerns maternal nutrition during pregnancy and its possible relation to early child development. ${ }^{325758}$

\section{Parental mental health in the transition to parenthood}

First, we included an examination of the course of depressive symptoms in the antenatal and postnatal period among subgroups of women. ${ }^{54}$ We found that maternal antenatal and postnatal depressive symptoms do not follow a uniform course; results rather support a model of several distinct trajectories of depressed mood associated with different adverse psychosocial and relational factors. Four different symptom trajectories were identified: only in pregnancy, only post partum, moderate and persistent up to 12 months post partum. Both researchers and clinicians should be aware of possible multiple courses of depressive mood and the risk factors behind these different trajectories, in order to better target intervention efforts in the perinatal period. Moreover, we have examined the association between adverse childhood experiences and symptoms of antenatal depression and anxiety in prospective fathers. ${ }^{22}$ Fathers who reported several such experiences had more depressive symptoms and anxiety before birth of the child than those with lower scores. We also found that paternal symptoms of anxiety and depression during pregnancy as well as their own adverse childhood experiences predicted stress and a negative perception of the infants' behaviour. ${ }^{55}$ In a similar vein, we investigated the multiple determinants of mothers' parenting stress and found that partner attachment style assessed prenatally was a salient predictor of such stress 12 months after birth. ${ }^{18}$ We further found a link between maternal adverse childhood experiences and later parenting stress, and showed that partner attachment style operated as a mediator in this link. Perceived difficult infant temperament also contributed to the experience of parenting stress in the mothers. Altogether, these results point to the clinical importance of paying attention to both parents' mental health during pregnancy. It is especially important to recognise the role of parental adverse childhood experiences in determining later parenting stress, and the need to help parents with a background of difficult relational experiences and an unsecure couple relationship before childbirth.

\section{Child development, infant mental health and early detection of infants at risk}

So far, four studies have been directed towards early child development and infant mental health. In one study, we found that parental perinatal depressive symptoms predicted child social-emotional difficulties and language delay at infant age 18 months. ${ }^{23}$ A differential effect, linking maternal symptoms to compromised socialemotional outcomes in children and paternal symptoms to poorer language outcomes was found. Perinatal depressive symptoms in both mothers and fathers may have a wide impact on child development, underscoring the importance of being aware of depression in fathers as well as in mothers in the perinatal period. It should be noted that a majority of Norwegian children between 1 and 2 years of age are cared for in professional childcare during the day $(83.5 \%) .{ }^{59}$ Possible stress reactions among the youngest children in connection with childcare has been of concern. We therefore studied the change of morning to mid-afternoon levels of the stress hormone cortisol at home and in childcare among toddlers. An increase in cortisol levels during the day in childcare compared with home was found. Of special interest is the finding that longer hours (8-9 per day) were associated with a greater increase in cortisol levels among the children compared with shorter hours (5-7), suggesting that separation from parents and interaction with several caregivers and children during the day may be especially demanding for toddlers who spend the longest days in childcare. ${ }^{52}$

In order to detect early deviance and to provide help to infants at risk for social-emotional difficulties as early as possible, it is important that assessment instruments are developmentally sensitive. To this aim, we investigated if the ITSEA scale could be reliably used as a screening instrument for children as young as 12 months. ${ }^{51}$ Results showed that clinically important social emotional problems and competence delays could be reliably detected even at ITSEA's lowest age limit. We also examined the 
applicability of a temperament questionnaire constructed for intervention. ${ }^{56}$ The temperamental dimensions of adaptability, persistence and regularity had coherent factor structures. The inclusion of concepts related to individual differences in infant response tendencies and regulatory efforts when assessing infant behaviour may broaden the understanding of parent-infant transactions, and can provide a valuable toolkit for individualised parent guidance at an early age.

\section{Maternal nutrition during pregnancy and later child development}

So far, three studies of maternal and child nutrition and later child development have been published. ${ }^{32} 5758$ First, we asked whether the pregnant women had sufficient iodine intake according to WHO criteria. ${ }^{57}$ In many cases, the women's diet did not secure a sufficient iodine intake. Second, mild-to-moderate maternal iodine deficiency in pregnancy was found to be associated with lower child language skills up to 18 months. ${ }^{32}$ Third, the iodine status and dietary iodine sources were studied cross-sectionally among the toddlers. ${ }^{57}$ Results showed that the iodine status among the children from different geographic areas in Norway was sufficient, indicated by a median UIC above the WHO cut-off of $100 \mu \mathrm{g} / \mathrm{L}$. To our knowledge, this is the largest and most complete study to date of maternal iodine status during pregnancy and its association with repeated measures of clinically assessed infant and toddler neurodevelopment. Preventing mildto-moderate iodine deficiency in pregnant women by securing adequate iodine status before conception is an optimal strategy to counteract the detrimental effects of inadequate iodine intake.

\section{Strengths and limitations}

This study has a cross-disciplinary nature with a frequent and in-depth assessment of mothers, fathers and their children from early in pregnancy. The use of a multimethod and multi-informant design including biological sampling, direct observation of behaviour, assessment of the children's development and self-reported information from mothers and fathers is a main strength. Moreover, well-validated measures have been used to assess both child development, child socio-emotional functioning and parental symptoms of depression, anxiety, adult attachment style and parenting stress.

The use of many repeated assessments may increase the odds for identifying developmental pathways and contributing parental and child risk and promoting factors. However, the LiN cohort alone may be underpowered when examining risk factors of outcomes with low prevalence. National as well as international collaboration is therefore encouraged with the aim of increasing sample size in connection with targeted research questions.

Furthermore, the parents who took part tended to have a higher educational level than was common in the general population at the different sites. The impact of educational level might be different if it had been more in line with that of the local populations. A related concern is selective attrition, as dropout was shown to be related to depressive symptoms. ${ }^{23}{ }^{54}$ However, appropriate statistical methods have been used to minimise the effect of selective attrition in papers based on this study population. ${ }^{18} 22235455$ In publications where multiple assessments over time have been included, based on both mother, father, and in some instances child data, we have used a structural equation (SEM) framework. ${ }^{18} 222354$ In these papers using longitudinal data from the study, full information maximum likelihood (FIML) has been used to reduce bias relating to missing data, including late recruitment, intermittent missingness and study dropout. FIML is regarded as a state-of-the-art missing data technique, providing unbiased parameter estimates under the missing at random (MAR) assumptions while at the same time providing high statistical power of the analyses. ${ }^{60} \mathrm{In}$ another paper, we used mixed effects models that gives valid results under the MAR assumption for the repeated measurements. ${ }^{55}$ Several assessments were based on selfreport. This applies to our measures of adverse childhood experiences, depressive and anxious symptoms, as well as attachment style. Hence, associations might be inflated due to shared methods variance.

\section{COLLABORATION}

The study is located at the Department of Psychology at the University of Oslo. Collaboration is encouraged. Further information and requests for collaboration can be obtained by contacting the principal investigator Vibeke Moe: vibeke.moe@psykologi.uio.no. The Institute of Marine Research (IMR), Bergen, and Uni Research Centre for Child and Youth Mental Health and Child Welfare, Bergen, and the Regional Centre for Child and Adolescent Mental Health, Eastern and Southern Norway are all collaborating institutions.

Acknowledgements The authors would like to thank the parents and the children who took part in this study for their generous willingness to share information with them. The authors would also like to thank the public health nurses who did the exacting job of collecting the data at the local sites.

Contributors VM and LS planned and developed the Little in Norway study design. MK, LD, MWM and KMS planned the nutrition part of the study. VM, EF, MK, LD, MWM, KMS, TvS, KSO, UTV and LS all contributed in planning of the design of the present paper. EF and VM performed analysis of data. VM took the main responsibility for drafting the article, while EF, MK, LD, MWM, KMS, TvS, KSO, UTV and LS contributed substantially in revising it critically for important intellectual content. All authors approved the final draft for publication.

Funding The Little in Norway study has been supported by grants from the Research Council of Norway (Grant 196156); the Regional Centre for Child and Adolescent Mental Health, Eastern and Southern Norway, the Department of Psychology, University of Oslo, Norway and the Norwegian Seafood Research Fund (FHF) (900842.FINS). TvS' contribution has been supported by a grant from the Research Council of Norway (Grant 288083).

Competing interests None declared.

Patient consent for publication Not required.

Ethics approval All procedures were in accordance with the ethical standards of the institutional and national research committee and with the 1964 Helsinki Declaration and its later amendments. The study protocol and the assessment procedures were reviewed and approved by the Norwegian Regional Committees for Medical and Health Research Ethics (reference number 2011/560). 
Provenance and peer review Not commissioned; externally peer reviewed.

Data availability statement Data are available on reasonable request.

Open access This is an open access article distributed in accordance with the Creative Commons Attribution Non Commercial (CC BY-NC 4.0) license, which permits others to distribute, remix, adapt, build upon this work non-commercially, and license their derivative works on different terms, provided the original work is properly cited, appropriate credit is given, any changes made indicated, and the use is non-commercial. See: http://creativecommons.org/licenses/by-nc/4.0/.

\section{ORCID iD}

Vibeke Moe http://orcid.org/0000-0002-8436-8341

\section{REFERENCES}

1 Räikkönen K, Pesonen A-K, Roseboom TJ, et al. Early determinants of mental health. Best Pract Res Clin Endocrinol Metab 2012;26:599-611.

2 Sameroff A. The transactional model. In: Sameroff A, ed. The transactional model of development: How children and contexts shape each other. Amer Psychol Assoc, 2009: 3-21.

3 Fox SE, Levitt P, Nelson III CA. How the timing and quality of early experiences influence the development of brain architecture. Child Dev 2010;81:28-40.

4 Scott S. Parenting quality and children's mental health: biological mechanisms and psychological interventions. Curr Opin Psychiat 2012;25:301-6.

5 Skovgaard AM, Olsen EM, Christiansen E, et al. Predictors (0-10 months) of psychopathology at age $1 \frac{1}{2}$ years - a general population study in The Copenhagen Child Cohort CCC 2000*. J Child Psychol \& Psychiat 2008;49:553-62.

6 Sameroff AJ, Seifer R, Zax M, et al. Early indicators of developmenta risk: Rochester longitudinal study. Schizophr Bull 1987;13:383-94.

7 Doyle O, Harmon CP, Heckman JJ, et al. Investing in early human development: timing and economic efficiency. Economics \& Human Biology 2009;7:1-6.

8 Nores M, Barnett WS. Benefits of early childhood interventions across the world: (under) investing in the very young. Econ Educ Rev 2010;29:271-82.

9 Odgers CL, Jaffee SR. Routine versus catastrophic influences on the developing child. Annu Rev Public Health 2013;34:29-48.

10 Felitti VJ, Anda RF. The relationship of adverse childhood experiences to adult medical disease, psychiatric disorders, and sexual behavior: Implications for healthcare. In: Lanius RA, Vermetten $\mathrm{E}$, eds. The impact of early life trauma on health and disease: the hidden epidemic. Cambridge Univ Press, 2010: 77-87.

11 Dube SR, Felitti VJ, Dong M, et al. The impact of adverse childhood experiences on health problems: evidence from four birth cohorts dating back to 1900. Prev Med 2003;37:268-77.

12 Chapman DP, Whitfield CL, Felitti VJ, et al. Adverse childhood experiences and the risk of depressive disorders in adulthood. $J$ Affect Disord 2004;82:217-25.

13 Felitti VJ, Anda RF, Nordenberg D, et al. Relationship of childhood abuse and household dysfunction to many of the leading causes of death in adults: the adverse childhood experiences (ACE) study. Am J Preventive Med 1998;1998:245-58.

14 McCarthy G, Maughan B. Negative childhood experiences and adult love relationships: the role of internal working models of attachment. Attach Hum Dev 2010;12:445-61.

15 McCarthy G, Taylor A. Avoidant/ambivalent attachment style as a mediator between abusive childhood experiences and adult relationship difficulties. J Child Psychol Psychiatry 1999;40:465-77.

16 Murphy A, Steele M, Dube SR, et al. Adverse childhood experiences (ACEs) questionnaire and adult attachment interview (AAl): implications for parent child relationships. Child Abuse Negl 2014;38:224-33.

17 Steele H, Bate J, Steele M, et al. Adverse childhood experiences, poverty, and parenting stress. Can J Behav Sci 2016;48:32-8.

18 Moe V, von Soest T, Fredriksen E, et al. The multiple determinants of maternal parenting stress 12 months after birth: the contribution of antenatal attachment style, adverse childhood experiences, and infant temperament. Front Psychol 2018;9.

19 Goodman SH, Gotlib IH. Risk for psychopathology in the children of depressed mothers: a developmental model for understanding mechanisms of transmission. Psychol Rev 1999;106:458-90.

20 Stein A, Pearson RM, Goodman SH, et al. Effects of perinatal mental disorders on the fetus and child. Lancet 2014;384:1800-19.

21 Ramchandani P, Psychogiou L. Paternal psychiatric disorders and children's psychosocial development. Lancet 2009;374:646-53. (2009).
22 Skjothaug T, Smith L, Wentzel-Larsen T, et al. Prospective fathers' adverse childhood experiences, pregnancy-related anxiety, and depression during pregnancy. Infant Ment Health J 2015;36:104-13.

23 Fredriksen E, von Soest T, Smith L, et al. Parenting stress plays a mediating role in the prediction of early child development from both parents' perinatal depressive symptoms. J Abnorm Child Psychol 2018.

24 King JC. The risk of maternal nutritional depletion and poor outcomes increases in early or closely spaced pregnancies. J Nutr 2003;133:1732S-6.

25 Wu G, Bazer FW, Cudd TA, et al. Maternal nutrition and fetal development. J Nutr 2004;134:2169-72.

26 Otto SJ, de Groot RHM, Hornstra G. Increased risk of postpartum depressive symptoms is associated with slower normalization after pregnancy of the functional docosahexaenoic acid status. Prostaglandins Leukot Essent Fatty Acids 2003;69:237-43.

27 Freeman MP, Hibbeln JR, Wisner KL, et al. Randomized doseranging pilot trial of omega-3 fatty acids for postpartum depression. Acta Psychiatr Scand 2006;113:31-5.

28 Hibbeln JR. Seafood consumption, the DHA content of mothers' milk and prevalence rates of postpartum depression: a cross-national, ecological analysis. J Affect Disord 2002;69:15-29.

29 Markhus MW, Skotheim S, Graff IE, et al. Low omega-3 index in pregnancy is a possible biological risk factor for postpartum depression. PLoS One 2013;8:e67617.

30 Abel MH, Caspersen IH, Meltzer HM, et al. Suboptimal maternal iodine intake is associated with impaired child neurodevelopment at 3 years of age in the Norwegian mother and child cohort study. $J$ Nutr 2017;147:1314-24.

31 Bath SC, Steer CD, Golding J, et al. Effect of inadequate iodine status in UK pregnant women on cognitive outcomes in their children: results from the Avon longitudinal study of parents and children (ALSPAC). Lancet 2013;382:331-7.

32 Markhus M, Dahl L, Moe V, et al. Maternal iodine status is associated with offspring language skills in infancy and toddlerhood. Nutrients 2018;10:1270.

33 Statistics Norway, 2019. Available: https://www.ssb.no/statbank/ table/12191/ [Accessed 02 Sep 2019].

34 Cox JL, Holden JM, Sagovsky R. Detection of postnatal depression: development of the 10-item Edinburgh postnatal depression scale. Brit J Psychiat 1987;150:782-6.

35 Russell M. New assessment tools for risk drinking during pregnancy: T-ACE, TWEAK, and others. Alcohol Res Health 1994;18:55-61.

36 Abidin RR. Parenting stress index. In: Professional manual. 3rd edn. Odessa, TX: Psychol Assessment Resources, Inc, 1995.

37 Brennan KA, Clark CL, Shaver PR. Self-report measurement of adult attachment: an integrative overview. In: Simpson JA, Rholes WS, eds. Attachment theory and close relationships. New York NY: Guilford, 1998: 46-76.

38 DeOliveira CA, Moran G, Pederson DR. Understanding the link between maternal adult attachment classifications and thoughts and feelings about emotions. Attach Hum Dev 2005;7:153-70.

39 Crugnola CR, Gazzotti S, Spinelli M, et al. Maternal attachment influences mother-infant styles of regulation and play with objects at nine months. Attach Hum Dev 2013;15:107-31.

40 Huizink AC, Mulder EJH, Robles de Medina PG, et al. Is pregnancy anxiety a distinctive syndrome? Early Hum Dev 2004;79:81-91.

41 Murray D, Cox JL. Screening for depression during pregnancy with the Edinburgh depression scale (EDDS). J Reprod Infant Psychol 1990;8:99-107.

42 Matthey S, Barnett B, Kavanagh DJ, et al. Validation of the Edinburgh postnatal depression scale for men, and comparison of item endorsement with their partners. J Affect Disord 2001;64:175-84.

43 Dahl L, Mæland CA, Bjørkkjær T. A short food frequency questionnaire to assess intake of seafood and $n-3$ supplements: validation with biomarkers. Nutr J 2011;10:127.

44 Markhus MW, Graff IE, Dahl L, et al. Establishment of a seafood index to assess the seafood consumption in pregnant women. Food Nutr Res 2013;57.

45 Field TM, Dempsey JR, Hallock NH, et al. The mother's assessment of the behavior of her infant. Infant Behavior and Development 1978;1:156-67

46 Bayley N. Bayley scales of infant and toddler development. Bayley-III: Harcourt Assessment, Psych. Corporation, 2006.

47 Guedeney A, Fermanian J. A validity and reliability study of assessment and screening for sustained withdrawal reaction in infancy: the alarm distress baby scale. Infant Ment Health $J$ 2001;22:559-75.

48 Cameron JR, Rice DC, Sparkman G, et al. Childhood temperamentbased anticipatory guidance in an HMO setting: a longitudinal study. J Community Psychol 2013;41:236-48. 
49 Thomas A, Chess S. The New York longitudinal study: From infancy to early adult life. In: Plomin R, Dunn J, eds. The study of temperament: changes, continuities, and challenges. Erlbaum, 1986: 39-52.

50 Carter AS, Briggs-Gowan MJ, Jones SM, et al. The Infant-Toddler social and emotional assessment (ITSEA): factor structure, reliability, and validity. J Abnorm Child Psychol 2003;31:495-514.

51 Sanner N, Smith L, Wentzel-Larsen T, et al. Early identification of social-emotional problems: applicability of the Infant-Toddler social emotional assessment (ITSEA) at its lower age limit. Infant Behavior and Development 2016;42:69-85.

52 Drugli MB, Solheim E, Lydersen S, et al. Elevated cortisol levels in Norwegian toddlers in childcare. Early Child Dev Care 2018;188:1684-95.

53 Wikenius E, Myhre AM, Page CM, et al. Prenatal maternal depressive symptoms and infant DNA methylation: a longitudinal epigenomewide study. Nord J Psychiatry 2019;73:257-63.

54 Fredriksen E, von Soest T, Smith L, et al. Patterns of pregnancy and postpartum depressive symptoms: latent class trajectories and predictors. J Abnorm Psychol 2017;126:173-83.
55 Skjothaug T, Smith L, Wentzel-Larsen T, et al. Does fathers' prenatal mental health bear a relationship to parenting stress at 6 months? Infant Ment Health J 2018;39:537-51.

56 Olafsen KS, Ulvund SE, Torgersen AM, et al. Temperamental adaptability, persistence, and regularity: parental ratings of Norwegian infants aged 6 to 12 months, with some implications for preventive practice. Infant Ment Health $J$ 2018;39:183-97.

57 Dahl L, Wik Markhus M, Sanchez P, et al. lodine deficiency in a study population of Norwegian pregnant Women-Results from the little in Norway study (Lin). Nutrients 2018;10:513.

58 Aakre I, Markhus MW, Kjellevold M, et al. Sufficient iodine status among Norwegian toddlers 18 months of age: crosssectional data from the little in Norway study. Food Nutrit Res 2018;2018:1443-52.

59 Statistics Norway, 2019. Available: https://www.ssb.no/utdanning/ statistikker/barnehager [Accessed 06 Sept 2019].

60 Graham JW. Missing data analysis: making it work in the real world. Annu Rev Psychol 2009;60:549-76. 